\section{Anticonceptivo de elección post hemorragia de la primera mitad del embarazo que terminaron en aborto}

\section{Post-hemorrhage contraceptive choice in the first half of pregnancy that ended in abortion}

\section{Contraceptivo de escolha pós-hemorrágica na pri- meira metade da gravidez que termina em aborto}

\section{Resumen}

Introducción: La planificación familiar y los anticonceptivos reducen los embarazos no deseados y así disminuyen la incidencia de abortos en especial los de alto riesgo. Se está intentando unificar los criterios de elección del método anticonceptivo, no existe uno ideal para todas las mujeres, el mejor será aquel que la mujer o de preferencia ambos miembros de la pareja elijan. El objetivo del estudio es; determinar la elección del método anticonceptivo posterior a hemorragia de la primera mitad del embarazo que terminaron en aborto. Metodología: El estudio fue descriptivo, transversal, observacional y retrospectivo. Realizado en Centro de Salud Sexual y Reproductiva CIES Cochabamba. La muestra fue compuesta por 200 expedientes clínicos de mujeres que realizaron consulta ginecológica, en el período de mayo a diciembre del 2019. Resultados: El grupo de pacientes que acudió al servicio con el diagnostico fueron: $28 \%$ de 16 a 20 años, $23 \%$ de 21 a 25 años, $18 \%$ de 26 a 30 años, $16 \%$ de 31 a 35 años, $13 \%$ de 36 a 40 años y 2\% de 41 a 46 años. Los métodos anticonceptivos elegidos por las usuarias fueron: 54\% implante subdérmico, $23 \%$ Inyectable, $12 \%$ píldora y $11 \% \mathrm{~T}$ de Cobre. Discusión: Se observó en el estudio, el grupo de mujeres en edad fértil que presento mayor incidencia de hemorragia de la primera mitad del embarazo con pérdida de producto fue entre los 16 a 20 años y el anticonceptivo de mayor aceptación fue el implante subdérmico, seguido de la píldora anticonceptiva.

Palabras claves: Anticonceptivos, Conducta de Elección, Hemorragia Uterina, Aborto.

\section{Summary}

Introduction: Family planning and contraceptives reduce unwanted pregnancies and thus reduce the incidence of abortions, especially in high-risk abortions. Attempts are being made to unify the contraceptive method choice criteria, there is no an ideal one for all women, the best one will be that which the woman or preferably both members of the couple choose. The study objective is; To determine the contraceptive method chosen after
Fuantina Ugarte Vasquez. ${ }^{1}$ Joseth Trujillo Vale. ${ }^{2}$ Marlon Santos Da Costa. ${ }^{2}$ Graciela Sara Villarroel Revollo. $^{3}$

Jacqueline Claure Salinas. ${ }^{4}$ (D)

Correspondencia a:

${ }^{1}$ Ginecologa Obstetra. Gerente regional CIES Cochabamba - Bolivia.

${ }^{2}$ Centro de Investigación Medicina UNITEPC.

"CIMU".

Cochabamba - Bolivia

${ }^{3}$ Licenciada en Enfermería.

Docente carrera de enfermería Universidad

Técnica Privada Cosmos UNITEPC.

Cochabamba - Bolivia

${ }^{4}$ Ginecologa Obstetra Hospital Mexico Sacaba, Ginecologa Colposcopia CIES, Docente de pregrado UMSS, Jefe de Residentes en Hosp Mexico.

Cochabamba - Bolivia

Email de contacto: fugarte@cies.org.bo chelylla1986@gmail.com jaque80273@hotmail.com

Procedencia y arbitraje: No comisionado, sometido a arbitraje externo

Recibido para publicación: 26 de enero del 2020 Aceptado para publicación: 27 de marzo del 2020

\section{Citar como:}

Ugarte Vasquez F, Trujillo Vale J, Santos Da Costa M, Villarroel Revollo GS, Claure Salinas J. Anticonceptivo de elección post hemorragia de la primera mitad del embarazo que terminaron en aborto. Re Ci Sa UNITEPC. 2020;7(1):25-31.

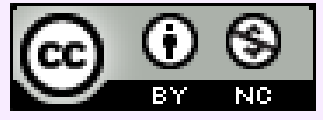

Esta obra está bajo una Licencia Creative Commons Atribución-NoComercial 4.0 internacional 
bleeding in the first half of pregnancy that ended in abortion. Methodology: The study was descriptive, cross-sectional, observational and retrospective. Carried out at the CIES Cochabamba Sexual and Reproductive Health Center. The sample was made up by 200 clinical women records who carried out a gynecological consultation, from May to December 2019. Results: The group of patients who came to the service with the diagnosis were: a $28 \%$ from 16 to 20 years old, a $23 \%$ from 21 to 25 years old, a $18 \%$ from 26 to 30 years old, a 16\% from 31 to 35 years old, a 13\% from 36 to 40 years old and a $2 \%$ from 41 to 46 years old. The contraceptive methods chosen by the users were: a $54 \%$ subdermal implant, a $23 \%$ injectable, a $12 \%$ pill and a $11 \%$ copper T. Discussion: In the study it was observed that the group of childbearing women age presented the highest incidence of hemorrhage in the first half of pregnancy with product lossing was between 16 to 20 years old and the most accepted contraceptive was the subdermal implant, followed of the contraceptive pill.

Keywords: Contraceptive Agents, Choice Behavior, Uterine Hemorrhage, Abortion.

\section{Resumo}

Introdução: O planejamento familiar e os contraceptivos reduzem gravidez indesejadas e, assim, reduzem a incidência de abortos, principalmente os de alto risco. Estão sendo feitas tentativas para unificar os critérios de escolha do melhor método contraceptivo; atualmente não existe um ideal para todas as mulheres; o melhor será aquele que a mulher ou de preferência, o casal escolherem. O objetivo do estudo é: Determinar a escolha do método contraceptivo após o sangramento na primeira metade da gravidez que terminou em aborto. Metodologia: $O$ estudo foi descritivo, transversal, observacional e retrospectivo. Realizado no Centro de Saúde Sexual e Reprodutiva do CIES Cochabamba. A amostra foi composta por 200 prontuários de mulheres que realizaram consulta ginecológica, no período de maio a dezembro de 2019. Resultados: O grupo de pacientes que compareceram ao serviço com o diagnóstico foram: $28 \%$ de 16 a 20 anos, $23 \%$ de 21 a 25 anos, $18 \%$ de 26 a 30 anos, $16 \%$ de 31 a 35 anos, $13 \%$ de 36 a 40 anos e $2 \%$ de 41 a 46 anos. Os métodos contraceptivos escolhidos pelos usuários foram: $54 \%$ implante subdérmico, $23 \%$ injetável, $12 \%$ comprimido e $11 \%$ cobre T. Discussão: Observou-se no estudo que o grupo de mulheres em idade fértil que apresentou maior incidência de sangramento na primeira metade da gravidez com perda de produto tinha entre 16 e 20 anos e o contraceptivo mais aceito foi o implante subdérmico, seguido da pílula contraceptiva.

Palavras chave: Anticoncepcionais, Comportamento de Escolha, Hemorragia Uterina, Aborto.

\section{Introducción.}

En conjunto la planificación familiar y los anticonceptivos reducen los embarazos no deseados y así disminuyen la incidencia de abortos en especial los de alto riesgo, en este sentido la Organización Mundial de la Salud viene realizando el esfuerzo desde hace más de dos décadas, de unificar los criterios de elección del método anticonceptivo para su uso (1-3).

Organizaciones mundiales describen que la planificación familiar no solo es un argumento de derechos humanos; sino que también es esencial para el empoderamiento de las mujeres, disminución de la pobreza y lograr el desarrollo sostenible (4). 
Promover el uso de métodos anticonceptivos durante la edad reproductiva es primordial. No existe un método ideal para todas las mujeres, el mejor método será aquel que la mujer o de preferencia ambos miembros de la pareja elijan, después de haber recibido una completa, detallada información y sean sometidos a una anamnesis detallada, pues estos no solo van a cumplir una función de prevención del embarazo, sino también la de prevenir el contagio de una enfermedad de transmisión sexual (5).

Los métodos anticonceptivos deben ser propuestos a las usuarias considerando los criterios de selección respecto al diagnóstico, ya que se debe considerar una serie de situaciones y entre ellas están las hemorragias con abortos (6).

La hemorragia de la primera mitad del embarazo, se define como la presencia de sangrado transvaginal causado por: aborto incompleto, huevo anembrionado, huevo muerto retenido, embarazo ectópico, embarazo molar y amenaza de aborto e interrupción del embarazo antes de las 22 semanas de gestación, con o sin expulsión del producto de la concepción que pone en riesgo la vida de la madre (6).

Para la salud publica los embarazos no planificados continúan siendo un problema, los datos indican que anualmente existen 74 millones de estos; 25 millones terminan en abortos peligrosos y 47000 mujeres mueren por esta causa en países en vías de desarrollo (7).

Después de significativos cambios en las políticas de población y la expansión de programas de planificación familiar en México, durante 1976 el 30\% de mujeres casadas utilizaban anticonceptivos y para el 1999 el $70 \%$ (8).

La organización mundial de la salud, público un estudio de Filipinas que reporta, cada año casi 2 millones de embarazos no planificados con más de 600.000 abortos peligrosos y que solo el 3\% de mujeres recibieron asesoramiento anticonceptivo (7).

Países con bajos ingresos económicos presentan: 300 millones de mujeres que usan anticonceptivos, el cual evitó más de 82 millones de embarazos no deseados, 25 millones de abortos y 125000 defunciones maternas para el 2016 (9).

En Bolivia, para el año 2018 presenta los siguientes datos; mujeres del grupo etario de 20 a 24 años del área rural presento embarazo antes de los 20 años en un 55\%, en comparación con el área urbana que llegó a 28,1\% (10).

El médico tiene un rol determinante en aproximar estos criterios a la realidad de cada una de sus pacientes, de forma que se consiga mostrar el mejor método disponible en cada caso, siempre brindando la información adecuada y respetando las opiniones y deseos de cada mujer o pareja. Para aminorar el elevado número de embarazos no planificados y abortos peligrosos que se producen en muchos países (11).

Por todo lo citado, el objetivo del estudio es; determinar la elección del método anticonceptivo posterior a hemorragia de la primera mitad del embarazo que terminaron en aborto, en mujeres que acuden al Centro de Salud Sexual y Reproductiva CIES Cochabamba.

\section{Metodología}

El estudio es descriptivo, de corte transversal, observacional y retrospectivo. Realizado en Centro de Salud Sexual y Reproductiva CIES Cochabamba. 
CIES es una organización de desarrollo social privado sin fines de lucro que tiene la misión de contribuir al ejercicio de los derechos sexuales y reproductivos, para mejorar la calidad de vida de la población boliviana urbana y rural, con énfasis en aquélla que vive en situaciones de vulnerabilidad (12).

La institución desarrolla el modelo de promoción y asistencia en salud sexual y reproductiva, que tiene un abordaje integral e innovador, con enfoque de género, generacional e intercultural (12).

La muestra fue compuesta por 200 expedientes clínicos de mujeres que realizaron consulta ginecológica, en el período de mayo a diciembre del 2019, se consideró una seguridad del $95 \%$ con una precisión $\mathrm{d}=0.0049 \%$, calculados por medio de fórmula establecida para muestras finitas.

Las unidades de la muestra se eligieron en función a los criterios de inclusión que fueron todos los registros con datos completos, con el diagnostico de hemorragia de la primera mitad del embarazo y en su defecto los criterios de eliminación fueron registros incompletos o poco legibles del cuaderno de registro de datos.

Para el análisis de resultados del presente estudio se elaboró una matriz de información estructurada con variables que corresponden a; edad, número de historia clínica, método anticonceptivo elegido y diagnóstico. Se exploró los expedientes clínicos aleatoriamente y para procesar la información se utilizó el programa IBM SPSS Statistics.

Se protegió el principio de confidencialidad tomando en cuenta la identificación personal usando numeración codificada. Asimismo, no se diferenció a la población en estudio por otros factores que podrían interferir en la vida particular de las personas, recolectándose solo datos pertinentes para el mismo

\section{Resultados}

Los resultados de la revisión de expedientes clínicos mostraron: en el grafico 1 se observa el grupo de pacientes que acudieron al servicio de ginecología obstetricia con el diagnóstico de hemorragia de la primera mitad del embarazo que termino en aborto y el grafico 2 muestra los métodos anticonceptivos optados por las usuarias.

Grafico 1. Pacientes por grupo etario que acudieron al CíES con el diagnostico de sangrado de la primera mitad del embrazo que termino en aborto.

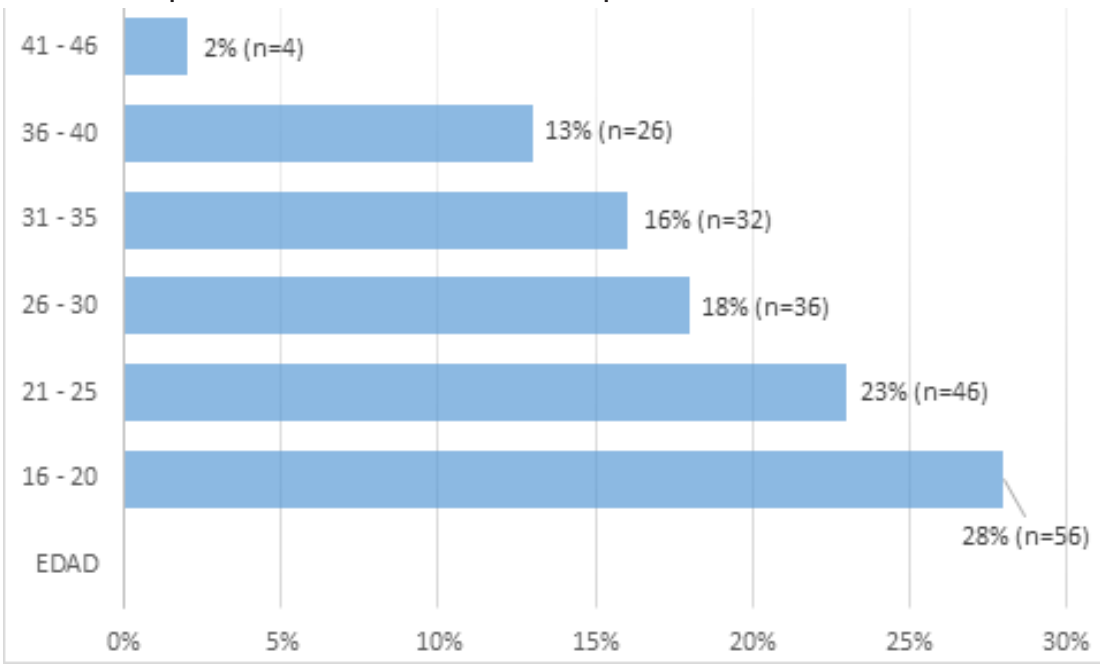

Fuente: Elaboración Propia 
Grafico 2. Métodos anticonceptivos elegidos por las usuarias del CíES después de sangrado de la primera mitad del embrazo que termino en aborto.

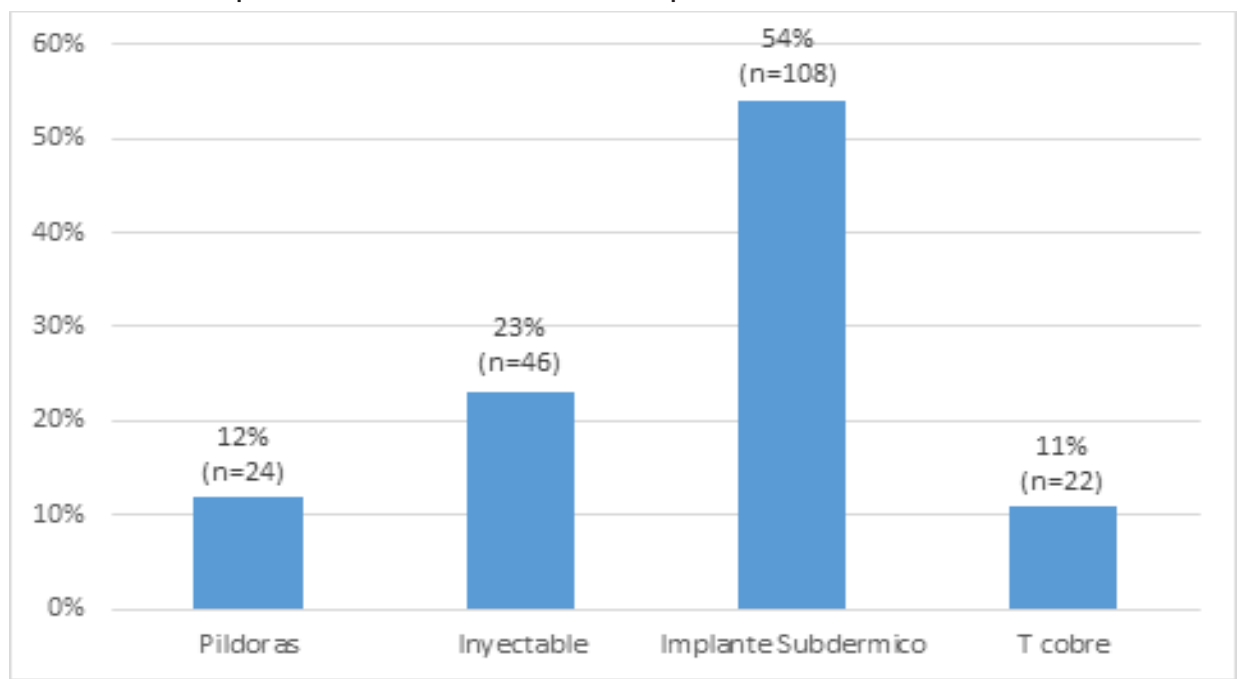

Fuente: Elaboración Propia

\section{Discusión}

La hemorragia de la primera mitad del embarazo que concluyeron con pérdida de producto es la complicación más frecuente de la gestación y un porcentaje elevado de mujeres tendrán algún tipo de aborto durante la edad reproductiva (13).

Este estudio expone que el rango de edad materna de 16 a 20 años, presento hemorragia de la primera mitad del embarazo en un $28 \%$. Este resultado comparando con otros estudios muestra a este grupo con mayor riesgo de sufrir esta patología y relacionado con el porcentaje es bajo en lo que se refiere al trabajo realizado en Venezuela que muestra 36,5\% (14).

El grupo etario de 21 a 25 años presento un 23\%, relacionando con el mismo estudio anteriormente citado el resultado es bajo, porque muestra $42 \%$. También esta descrito que las mujeres entre 20 a 24 años presentan tasa elevadas de aborto en relación a otros grupos etarios (15).

Todas las mujeres del estudio eligieron métodos anticonceptivos posterior a hemorragia de la primera mitad del embarazo, el que presento mayor aceptación fue el implante subdermico, que consiste en una varilla pequeña, que se aplica mediante una pequeña incisión en el brazo. Cada una dura tres años, tiempo en el que la mujer está protegida contra un embarazo no deseado con un 54\%, que es elevado en relación con otros estudios que muestran un $6 \%$ (16), y es la más aceptada por las mujeres en edad fértil de este este estudio.

Al implante subdermico le siguen; el inyectable trimestral con $23 \%$, píldoras anticonceptivas $12 \%$ y dispositivo intrauterino $11 \%$ estos valores son elevados en relación con el estudio citado previamente, porque en dicho trabajo muestra que el $83 \%$ de mujeres no eligieron ningún anticonceptivo.(16).

En conclusión se observó en el estudio, el grupo de mujeres en edad fértil que presento mayor incidencia de hemorragia de la primera mitad del embarazo con pérdida de producto fue entre los 16 a 20 años y el anticonceptivo de mayor aceptación fue el 
implante subdérmico.

\section{Referencias bibliográficas}

1. Organization WH. Criterios médicos de elegibilidad para el uso de anticonceptivos. 3. ${ }^{\text {a }}$ ed. World Health Organization; 2005. Ginebra.

2. Soriano Fernández H, Rodenas García L, Moreno Escribano D. Criterios de Elegibilidad de Métodos Anticonceptivos: Nuevas Recomendaciones. Rev Clínica Med Fam. 2010; 3(3):206-16.

3. OMS | Criterios médicos de elegibilidad para el uso de anticonceptivos [Internet]. WHO. World Health Organization; 2020 [citado 16 de enero de 2020]. Disponible en: http://www.who.int/reproductivehealth/publications/family_planning/Ex-SummMEC-5/es/

4. ONU. La planificación familiar es un derecho humano [Internet]. Noticias ONU. 2018 [citado 16 de enero de 2020]. Disponible en: https://news.un.org/es/ story/2018/07/1437611

5. Peláez Mendoza J. El uso de métodos anticonceptivos en la adolescencia. Rev Cuba Obstet Ginecol. 2016; 42(1):0-0.

6. Ministerio de Salud. Manual de Normas, Reglas, Protocolos y Procedimientos Técnicos para el Manejo de las Hemorragias de la Primera Mitad del Embarazo [Internet]. Ministerio de Salud y Deportes de Bolivia. Dirección General de Servicios de Salud. Unidad de Servicios de Salud y Calidad; 2009 [citado 17 de enero de 2020]. Disponible en: http://clacaidigital.info:8080/xmlui/handle/123456789/762

7. OMS. Un nuevo estudio de la OMS relaciona las altas tasas de embarazos no planificados con las deficiencias de los servicios de planificación familiar [Internet]. 2019 [citado 17 de enero de 2020]. Disponible en: https://www.who.int/es/newsroom/detail/25-10-2019-high-rates-of-unintended-pregnancies-linked-to-gaps-infamily-planning-services-new-who-study

8. Casique I. Uso de anticonceptivos en México: ¿qué diferencia hacen el poder de decisión y la autonomía femenina? Papeles Poblac. marzo de 2003;9(35):209-32.

9. Guevara-Ríos E. Impacto de la planificación familiar en la salud de la mujer. Rev Peru Investig Materno Perinat. 2017; 6(1):7-8.

10. INE. Embarazo y maternidad son mayores en área rural [Internet]. 2018 [citado 17 de enero de 2020]. Disponible en: https://www.ine.gob.bo/index.php/prensa/notas-de-prensa/item/3113-embarazo-y-maternidad-son-mayores-en-area-rural

11. Gutiérrez M. La planificación familiar como herramienta básica para el desarrollo. Rev Peru Med Exp Salud Pública. Julio de 2013; 30(3):465-70.

12. CIES. Salud Sexual Salud Reproductiva - Clínica CIES La Paz - Clínica CIES Cochabamba - Clínica CIES Santa Cruz [Internet]. 2020 [citado 17 de enero de 2020]. Disponible en: https://www.cies.org.bo/

13. Romero Guadix B, Martínez Navarro L, González Paredes A, Fontes Jiménez J. Epidemiología del aborto de repetición. Prog Obstet Ginecol. 2012; 55(7):312-20.

14. Pérez-Arciniegas E, Godoy-Albornoz D, Quiroz-Figuera D, Quiroz D, Tovar-Tho- 
mas C, Romero-Herrera A, et al. Aspectos clínico-epidemiológicos del aborto en un hospital de Upata. Estado Bolívar-Venezuela. 2016; 27(1):7.

15. Aborto a nivel mundial 2017-Resumen ejecutivo [Internet]. Guttmacher Institute. 2018 [citado 5 de julio de 2020]. Disponible en: https://www.guttmacher.org/es/ report/aborto-a-nivel-mundial-2017-resumen-ejecutivo

16. Valverde-Espinoza N, Barja-Ore J. Método anticonceptivo de elección en el postaborto. Ginecol Obstet México. 2019; 87(12):814-9. 